Pure and Applied Mathematics Quarterly

Volume 9, Number 1

(Special Issue: In honor of

Dennis Sullivan, Part 1 of 2)

49-71, 2013

\title{
Uniformly Perfect Domains and Convex Hulls: Improved Bounds in a Generalization of a Theorem of Sullivan
}

\author{
Martin Bridgeman and Richard D. Canary \\ Dedicated to Dennis Sullivan on the occasion of his 70th birthday
}

\begin{abstract}
Given a hyperbolic domain $\Omega$, the nearest point retraction is a conformally natural homotopy equivalence from $\Omega$ to the boundary $\operatorname{Dome}(\Omega)$ of the convex core of its complement. Marden and Markovic showed that if $\Omega$ is uniformly perfect, then there exists a conformally natural quasiconformal map from $\Omega$ to $\operatorname{Dome}(\Omega)$ which admits a bounded homotopy to the nearest point retraction. We obtain an explicit upper bound on the quasiconformal dilatation which depends only on the injectivity radius of the domain.
\end{abstract}

Keywords: Uniformly perfect domains, convex hulls and Kleinian groups.

\section{INTRODUCTION}

If $\Omega$ is a hyperbolic domain in the Riemann sphere $\hat{\mathbb{C}}$, then the boundary $\operatorname{Dome}(\Omega)$ of the convex core of its complement is a hyperbolic surface in its intrinsic metric $([12,24])$. The nearest point retraction $r: \Omega \rightarrow \operatorname{Dome}(\Omega)$, which maps a point $z \in \Omega$ to the unique point of intersection of the smallest horoball based at $z$ which intersects $\operatorname{Dome}(\Omega)$, gives a conformally natural homotopy equivalence. Sullivan $[23]$ (see also $[12,14]$ ) showed that there exists $K_{0}$ such that if $\Omega$ is simply connected, then $r$ is homotopic to a conformally natural $K_{0}$-quasiconformal

Received October 10, 2011.

Canary was partially supported by NSF grant DMS - 1006298. 
map. Marden and Markovic [18] showed that if $\Omega$ is uniformly perfect, then there is a conformally natural quasiconformal map from $\Omega$ to $\operatorname{Dome}(\Omega)$ which admits a bounded homotopy to the nearest point retraction and that the dilatation of the map may be bounded in terms of the injectivity radius of $\Omega$ in the Poincaré metric. (We recall that $\Omega$ is uniformly perfect if and only if there is a non-zero lower bound for the injectivity radius of $\Omega$ in the Poincaré metric.) In this paper, we combine the results of [6] and the techniques of Epstein-Marden-Markovic [14] to obtain explicit bounds on the quasiconformal dilatation. We further show that these bounds are close to optimal.

Theorem 1.1. There exists a function $M: \mathbb{R}_{+} \rightarrow \mathbb{R}_{+}$such that if $\Omega \subset \hat{\mathbb{C}}$ is uniformly perfect and $\nu>0$ is a lower bound for its injectivity radius in the Poincaré metric, then there is a conformally natural $M(\nu)$-quasiconformal map

$$
\phi: \Omega \rightarrow \operatorname{Dome}(\Omega)
$$

which admits a bounded homotopy to the nearest point retraction $r: \Omega \rightarrow \operatorname{Dome}(\Omega)$. Moreover,

$$
M(\nu)=48 \pi e^{m} e^{\pi^{2} / 2 \nu}+12 \pi \leq 2220 e^{\pi^{2} / 2 \nu}+38
$$

where $m=\cosh ^{-1}\left(e^{2}\right) \approx 2.689$.

We also obtain a version of our main theorem, where the quasiconformal dilatation is bounded by the injectivity radius of $\operatorname{Dome}(\Omega)$. We recall that $\Omega$ is uniformly perfect if and only if there is a positive lower bound for the injectivity radius of Dome $(\Omega)$ (see Bridgeman-Canary [5, Lemmas 8.1 and 9.1]).

Theorem 1.2. There exists a function $N: \mathbb{R}_{+} \rightarrow \mathbb{R}_{+}$such that if $\Omega \subset \hat{\mathbb{C}}$ is uniformly perfect and $\hat{\nu}>0$ is a lower bound for the injectivity radius of $\operatorname{Dome}(\Omega)$ in its intrinsic metric, then there is a conformally natural $N(\hat{\nu})$-quasiconformal map

$$
\phi: \Omega \rightarrow \operatorname{Dome}(\Omega)
$$

which admits a bounded homotopy to the nearest point retraction $r: \Omega \rightarrow \operatorname{Dome}(\Omega)$. Moreover,

$$
N(\hat{\nu})=\frac{24 \pi}{\hat{\nu}}+12 \pi
$$

We recall that a map $f: \Omega \rightarrow \mathbb{H}^{3}$ is said to be conformally natural if whenever $\Gamma$ is a group of conformal automorphisms of $\hat{\mathbb{C}}$ which preserve $\Omega$, then

$$
f(\gamma(z))=\gamma(f(z))
$$


for all $z \in \Omega$ and $\gamma \in \Gamma$ where on the right-hand side we have extended the conformal automorphism of $\hat{\mathbb{C}}$ to the associated isometry of $\mathbb{H}^{3}$.

If $N=\mathbb{H}^{3} / \Gamma$ is a hyperbolic 3-manifold, we let $\Omega(\Gamma)$ be the largest open set in $\hat{\mathbb{C}}$ which $\Gamma$ acts properly discontinuously on and consider the conformal boundary $\partial_{c} N=\Omega(\Gamma) / \Gamma$. We will assume that $\Gamma$ is not virtually abelian, which guarantees that $\Omega(\Gamma)$ is hyperbolic. The convex core $C(N)$ of $N$ is the quotient $C H(\hat{\mathbb{C}}-\Omega(\Gamma)) / \Gamma$ where $C H(\hat{\mathbb{C}}-\Omega(\Gamma))$ denotes the convex hull in $\mathbb{H}^{3}$ of $\hat{\mathbb{C}}-\Omega(\Gamma)$. Then

$$
\operatorname{Dome}(\Omega(\Gamma))=\partial C H(\hat{\mathbb{C}}-\Omega(\Gamma))
$$

and the nearest point retraction $r: \Omega(\Gamma) \rightarrow \operatorname{Dome}(\Omega(\Gamma))$ descends to a homotopy equivalence

$$
\bar{r}: \partial_{c} N \rightarrow \partial C(N)
$$

If $\Omega(\Gamma)$ is uniformly perfect, the conformally natural quasiconformal map

$$
\phi: \Omega(\Gamma) \rightarrow \operatorname{Dome}(\Omega(\Gamma))
$$

guaranteed by Theorem 1.1 descends to a quasiconformal map

$$
\bar{\phi}: \partial_{c} N \rightarrow \partial C(N)
$$

which admits a bounded homotopy to $\bar{r}$. We recall that $\Omega(\Gamma)$ is uniformly perfect whenever $\Gamma$ is finitely generated (see Pommerenke [21]).

Corollary 1.3. If $N=\mathbb{H}^{3} / \Gamma$ is a hyperbolic 3-manifold and $\nu>0$ is a lower bound for the injectivity radius of $\Omega(\Gamma)$, then there is a $M(\nu)$-quasiconformal map

$$
\bar{\phi}: \partial_{c} N \rightarrow \partial C(N)
$$

which admits a bounded homotopy to the nearest point retraction $\bar{r}$.

Moreover, if $\hat{\nu}>0$ is a lower bound for the injectivity radius of $\operatorname{Dome}(\Omega(\Gamma))$, then $\bar{\phi}$ is $N(\hat{\nu})$-quasiconformal.

It is instructive to consider the special case of a round annulus $\Omega(s)$ of modulus $\frac{s}{2 \pi}$. In section 5 , we observe that its minimal injectivity radius is given by $\nu(s)=\frac{\pi^{2}}{s}$ while the minimal injectivity radius of $\operatorname{Dome}(\Omega(s))$ is given by $\hat{\nu}(s)=\frac{\pi}{\sinh (s / 2)}$. The minimal quasiconformal dilatation of a quasiconformal map from $\Omega(s)$ to $\operatorname{Dome}(\Omega(s))$ is then given by

$$
K(s)=\frac{\pi \sinh (s / 2)}{s} \approx \frac{\nu(s) e^{\pi^{2} / 2 \nu(s)}}{2 \pi} \approx \frac{\pi^{2}}{2 \hat{\nu}(s) \log (1 / \hat{\nu}(s))}
$$


(where the approximations hold as $s \rightarrow \infty$ ). These examples indicate that our estimates "almost" have the correct asymptotic form.

In a final section, we obtain lower bounds on the quasiconformal constant when a uniformly perfect domain has "small" injectivity radius.

Proposition 6.1. Let $\Omega$ be a uniformly perfect domain in $\hat{\mathbb{C}}$. Suppose that $\phi: \Omega \rightarrow \operatorname{Dome}(\Omega)$ is a $K$-quasiconformal map which is homotopic to the nearest point retraction. If $\Omega$ contains a point of injectivity radius $\nu \in(0, .5)$, in the Poincaré metric, then

$$
K \geq \frac{\nu e^{\frac{\pi^{2}}{2 \sqrt{e} \nu}}}{\pi^{2} e^{\frac{\pi}{2}}}=O\left(\nu e^{\frac{\pi^{2}}{2 \sqrt{e} \nu}}\right)
$$

Acknowledgements: The authors would like to thank the referees for their careful reading of the paper and helpful suggestions.

\section{Historical Discussion}

The investigation of the relationship between the geometry of a domain and the boundary of the convex core of its complement was initiated by Dennis Sullivan. We take the occasion of Dennis' 70th birthday as an excuse to provide a partial tour of results inspired by and/or related to Sullivan's Theorem.

Sullivan's Theorem: ([23]) There exists $K_{0}>1$ such that if $N=\mathbb{H}^{3} / \Gamma$ is a hyperbolic 3-manifold with finitely generated fundamental group and each component of $\Omega(\Gamma)$ is simply connected, then the nearest point retraction is homotopic to a $K_{0}$-quasiconformal map

$$
\phi: \partial_{c} N \rightarrow \partial C(N)
$$

Sullivan used his theorem in his exposition of Thurston's proof that 3-manifolds fibering over the circle are geometrizable. (In fact, Sullivan only states his theorem in the setting of quasifuchsian hyperbolic 3-manifolds.) The key issue there was to establish a relationship between the geometry of the conformal boundary 
and the internal geometry of the hyperbolic 3-manifold. From this viewpoint, Sullivan's Theorem may be viewed as a vast generalization of a fundamental result of Bers.

Bers' Theorem: ([1, Theorem 3]) Suppose that $N=\mathbb{H}^{3} / \Gamma$ is a hyperbolic 3manifold with finitely generated fundamental group and each component of $\Omega(\Gamma)$ is simply connected. If $\alpha$ is a closed geodesic in $\partial_{c} N$ (in the Poincaré metric) and $\alpha^{*}$ is the geodesic in $N$ in the homotopy class of $\alpha$, then

$$
l_{N}\left(\alpha^{*}\right) \leq 2 l_{\partial_{c} N}(\alpha)
$$

where $l_{N}\left(\alpha^{*}\right)$ denotes the length of $\alpha^{*}$ in $N$ and $l_{\partial_{c} N}(\alpha)$ denotes the length of $\alpha$ in $\partial_{c} N$.

Bers also originally stated his result only in the setting of quasifuchsian hyperbolic 3-manifolds. We note that in many applications, Bers' result provides all the information one needs about the relationship between the geometry of the conformal boundary and the geometry of the hyperbolic 3-manifold.

Another generalization of Bers' Theorem is provided by Epstein, Marden and Markovic:

Theorem 2.1. (Epstein-Marden-Markovic [13, Theorem 3.1]) If $\Omega$ is a simply connected hyperbolic domain, then the nearest point retraction is 2-Lipschitz.

Bridgeman [4] showed that, in the setting of Sullivans' Theorem, the nearest point retraction $\bar{r}: \partial_{c} N \rightarrow \partial C(N)$ has a $\left(1+\frac{2 \pi}{\log (3)}\right)$-Lipschitz homotopy inverse. This paper also introduced the technique of bounding the total bending along an arc in the boundary of the convex hull which was used in many subsequent results. Bishop [2, Lemma 8] observed that $r$ is a quasi-isometry with uniform quasi-isometry constants when $\Omega$ is simply connected. Bridgeman and Canary [6] provide explicit bounds

Theorem 2.2. (Bridgeman-Canary [6]) If $\Omega$ is a simply connected hyperbolic domain, then the nearest point retraction is a $\left(L^{\prime}, L_{0}^{\prime}\right)$-quasi-isometry, where $L^{\prime} \approx$ 4.56 and $L_{0}^{\prime} \approx 8.05$.

Another generalization of Bers' result is given by the length bounds on curves developed in the solution of Thurston's Ending Lamination Conjecture, see $[20,8]$. 
These results also provide lower bounds on the length of closed geodesics in a quasifuchsian hyperbolic 3-manifold in terms of the geometry of the conformal boundary.

Epstein and Marden [12] published the first complete proof of Sullivan's Theorem and noted that its proof can be extended to the setting of simply connected hyperbolic domains in $\hat{\mathbb{C}}$. Moreover, they obtained the first explicit bounds on $K_{0}$.

Theorem 2.3. (Epstein-Marden [12]) If $\Omega$ is a simply connected, hyperbolic domain in $\hat{\mathbb{C}}$, then there exists a conformally natural $K_{0}$-quasiconformal map

$$
\phi: \Omega \rightarrow \operatorname{Dome}(\Omega)
$$

which admits a bounded homotopy to the nearest point retraction. Moreover,

$$
K_{0} \leq 82.7 .
$$

Thurston [26] asked whether or not one could choose $K_{0}=2$. Epstein, Marden and Markovic [13,14] showed that that the optimal value of $K_{0}$ lies between 2.1 and 13.88 (see also Komori-Matthews [17] for an explicit Kleinian group whose domain of discontinuity provides a counterexample to Thurston's $K=2$ question). Bishop [3] showed that if one does not require the quasiconformal map to be conformally natural, then one can bound the quasiconformal dilatation above by 7.82. Epstein and Markovic [15], showed that even if one removes the requirement that the quasiconformal map be conformally natural, one cannot always bound the quasiconformal dilatation above by 2 .

The first generalization of Bers' result to the setting of hyperbolic manifolds where the domain of discontinuity is not simply connected was provided by:

Theorem 2.4. (Canary [9]) There exists a function $R:(0, \infty) \rightarrow(0, \infty)$ such that if $N=\mathbb{H}^{3} / \Gamma$ is a hyperbolic 3-manifold, $\nu>0$ is a lower bound for the injectivity radius of $\Omega(\Gamma)$, and $\alpha$ is a closed geodesic in $\partial_{c} N$ of length $l(\alpha)$, then

$$
l_{N}\left(\alpha^{*}\right) \leq R(\nu) l(\alpha)
$$

where $R(\nu)=\max \left\{\sqrt{2}(k+\log 2), \sqrt{2}\left(\frac{k \nu+8 \pi k+2 \pi^{2}}{\nu}\right)\right\}$ and $k=4+\log (3+2 \sqrt{2})$.

Canary [10] later established bounds on lengths of curves in the boundary of the convex core without assuming that domain of discontinuity was uniformly perfect. 
Theorem 2.5. (Canary [10]) Suppose that $N$ is a hyperbolic 3-manifold and that $r: \partial_{c} N \rightarrow \partial C(N)$ is the nearest point retraction from its conformal boundary to the boundary of its convex core. If $\alpha$ is a closed geodesic in the conformal boundary of length $L$, then

$$
l_{\partial C(N)}\left(r(\alpha)^{*}\right)<45 L e^{\frac{L}{2}}
$$

where $l_{\partial C(N)}\left(r(\alpha)^{*}\right)$ denotes the length of the closed geodesic $r(\alpha)^{*}$ in the homotopy class of $r(\alpha)$ in the intrinsic metric on $\partial C(N)$.

Bridgeman and Canary [5] showed that if $\nu>0$ is a lower bound for the injectivity radius of $\Omega(\Gamma)$ and $\Gamma$ is finitely generated, then the nearest point retraction is $2 \sqrt{2}\left(k+\frac{\pi^{2}}{2 \nu}\right)$-Lipschitz. Moreover, the nearest point retraction has a $P(\hat{\nu})$-Lipschitz homotopy inverse where $P(\hat{\nu}) \approx \frac{2 \pi}{\hat{\nu}}$ as $\hat{\nu} \rightarrow 0$.

Marden and Markovic [18] established the first direct analogue of Sullivan's Theorem in the setting of uniformly perfect hyperbolic domains.

Theorem 2.6. (Marden-Markovic [18]) Suppose that $\Omega$ is a hyperbolic domain in $\hat{\mathbb{C}}$. There exists a conformally natural quasiconformal map $\phi: \Omega \rightarrow \operatorname{Dome}(\Omega)$ which admits a bounded homotopy to the nearest point retraction if and only if $\Omega$ is uniformly perfect. Moreover, the quasiconformal dilatation of $\phi$ may be bounded by some function of the injectivity radius of $\Omega$ in the Poincaré metric.

Marden and Markovic also showed that the nearest point retraction is a quasiisometry if and only if $\Omega$ is uniformly perfect. Again, the quasi-isometry constants depend on a lower bound for the injectivity radius. However, as their arguments make crucial use of compactness arguments, Marden and Markovic do not find explicit bounds on the quasiconformal constants or quasi-isometry constants in terms of the injectivity radius.

In an earlier paper [6], we showed:

Theorem 2.7. (Bridgeman-Canary [6]) If $\Omega$ is a uniformly perfect, hyperbolic domain in $\hat{\mathbb{C}}$ and $\nu>0$ is a lower bound on its injectivity radius, then the nearest point retraction is $2 \sqrt{2}\left(k+\frac{\pi^{2}}{2 \nu}\right)$-Lipschitz and is a $\left((2 \sqrt{2})\left(k+\frac{\pi^{2}}{2 \nu}\right), L_{0}\right)$ quasi-isometry, where $k=4+\log (3+2 \sqrt{2})$ and $L_{0} \approx 7.12$.

Theorem 2.7 is obtained as a corollary of a result which asserts that if we give any hyperbolic domain $\Omega$ the Thurston metric, then the nearest point retraction 
is 1-Lipschitz and a $\left(L, L_{0}\right)$-quasi-isometry where $L \approx 8.49$ and $L_{0} \approx 7.12$. As another corollary of this result, we show that the nearest point retraction is Lipschitz (with respect to the Poincaré metric on $\Omega$ ) if and only if $\Omega$ is uniformly perfect (which was originally conjectured by Marden and Markovic [18].)

We also showed that if $\Omega$ is uniformly perfect, $r$ lifts to a quasi-isometry between the universal covers of $\Omega$ and $\operatorname{Dome}(\Omega)$ (see Theorem 3.7). We combined these results with work of Douady-Earle [11] to obtain explicit bounds, in terms of $\nu$, on the quasiconformal dilatation of the conformally natural map between $\Omega$ and Dome $(\Omega)$. However, our earlier bounds were very far from optimal, and the results in this paper provide a substantial improvement.

\section{BACKGROUND}

In this section, we assemble the ingredients of our proof. Section 3.1 reviews the theory of pleated planes. Section 3.2 recalls basic properties of the nearest point retraction and uses work of Bridgeman-Canary [6] to give a bound on the "roundness" of the bending lamination of Dome $(\Omega)$. Section 3.3 recalls work of Epstein, Marden, and Markovic [13] on complex earthquakes which gives rise to a conformally natural quasiconformal map from $\Omega$ to $\operatorname{Dome}(\Omega)$ when the roundness of the bending lamination of $\operatorname{Dome}(\Omega)$ is "small." Section 3.4 recalls the complex angle scaling maps used by Epstein, Marden, and Markovic [14] to associate a family of quasiregular maps to a family of complex earthquakes.

3.1. Pleated planes. Throughout this paper we will use the disk model for $\mathbb{H}^{2}$ and identify $\partial_{\infty} \mathbb{H}^{2}$ with $\mathbb{S}^{1}$. Let $G\left(\mathbb{H}^{2}\right)$ be the set of unoriented geodesics on $\mathbb{H}^{2}$. We can identify $G\left(\mathbb{H}^{2}\right)=\left(\mathbb{S}^{1} \times \mathbb{S}^{1}-\Delta\right) / \mathbb{Z}_{2}$. A geodesic lamination on $\mathbb{H}^{2}$ is a closed subset of $G\left(\mathbb{H}^{2}\right)$ such that distinct geodesics in the subset do not intersect. A measured lamination $\mu$ on $\mathbb{H}^{2}$ is a non-negative measure on the space of geodesics $G\left(\mathbb{H}^{2}\right)$ supported on a geodesic lamination. The measure $\mu$ assigns a measure to any arc transverse to the support by restricting $\mu$ to the set of geodesics that intersect the arc.

In [13], Epstein, Marden and Markovic defined the roundness of a measured lamination $\mu$ to be

$$
\|\mu\|=\sup _{C} \mu(C)
$$


where the sup is taken over all open geodesic arcs of unit length which are transverse to $\mu$.

In general, if $S=\mathbb{H}^{2} / \Gamma$ is a hyperbolic surface, then a measured lamination $\mu$ on $S$ lifts to a measured lamination $\tilde{\mu}$ on $\mathbb{H}^{2}$ and we define the roundness

$$
\|\mu\|=\|\tilde{\mu}\| .
$$

Of course, $\|\mu\|$ can also be defined intrinsically.

Given a measured lamination $\mu$ on $\mathbb{H}^{2}$, one can define a convex pleated plane

$$
P_{\mu}: \mathbb{H}^{2} \rightarrow \mathbb{H}^{3}
$$

by fixing a component of the complement of $\mu$ and mapping all other components isometrically after bending along the support of $\mu$ by an amount given by the transverse measure on $\mu$ (see [12, Chapter 3]). The map $P_{\mu}$ is well-defined up to post-composition by an isometry. In the case when $\mu$ is a finite collection of geodesics with discrete measure, $P_{\mu}$ is defined by mapping each component of the complement of $\mu$ isometrically so that the the dihedral angle between any two adjacent components is given by the value of the measure on that geodesic. For the general case, one can define $P_{\mu}$ by considering a sequence $\left\{\mu_{n}\right\}$ of finiteleaved laminations converging to $\mu$ and letting $P_{\mu}$ be the limit of $\left\{P_{\mu_{n}}\right\}$ (see $[12$, Theorem 3.11.9]).

Epstein, Marden and Markovic [13] showed that the pleated plane is an embedding when the roundness is small.

Theorem 3.1. ([13, Theorem 4.2]) There exists a constant

$$
c_{2}>.73
$$

such that if $\mu$ is a measured lamination on $\mathbb{H}^{2}$ and $\|\mu\| \leq c_{2}$, then $P_{\mu}$ is a bilipschitz embedding.

Remark: Epstein and Jerrard (see [7]) proved that one can take $c_{2} \approx .948$ in Theorem 3.1.

3.2. The nearest point retraction and the bending lamination. Let $\Omega \subseteq$ $\mathbb{S}^{2}$ be a hyperbolic domain and let $\operatorname{Dome}(\Omega)$ be the boundary of the convex hull of $\hat{\mathbb{C}}-\Omega$. The nearest point retraction

$$
r: \Omega \rightarrow \operatorname{Dome}(\Omega)
$$


is a conformally natural homotopy equivalence which extends continuously to a map from $\bar{\Omega}$ to $\operatorname{Dome}(\Omega) \cup \partial \Omega$ which is the identity on $\partial \Omega$. In the special case that $\hat{\mathbb{C}}-\Omega$ lies in a round circle, $C H(\hat{\mathbb{C}}-\Omega)$ is a subset of a totally geodesic plane in $\mathbb{H}^{3}$ and one defines $\operatorname{Dome}(\Omega)$ to be the double of $C H(\hat{\mathbb{C}}-\Omega)$ along its boundary (as a surface in the plane). (See Epstein-Marden [12, Chapter 1] for an extensive discussion of the basic properties of the nearest point retraction.)

Thurston [24] observed that the intrinsic metric on $\operatorname{Dome}(\Omega)$ is a complete hyperbolic metric. Moreover, he showed that there exists a lamination $\mu$ on $\mathbb{H}^{2}$ such that Dome $(\Omega)=P_{\mu}\left(\mathbb{H}^{2}\right)$ and that $P_{\mu}: \mathbb{H}^{2} \rightarrow \operatorname{Dome}(\Omega)$ is a locally isometric covering map. Epstein and Marden [12, Chapter 1] developed this observation more fully, see especially sections 1.11 and 1.12 .

Lemma 3.2. If $\Omega$ is a hyperbolic domain, there is a lamination $\mu$ on $\mathbb{H}^{2}$ such that $P_{\mu}$ is a locally isometric covering map with image Dome $(\Omega)$.

The lamination $\mu$ descends to a lamination $\hat{\mu}$ on $\operatorname{Dome}(\Omega)$ which is called the bending lamination of $\operatorname{Dome}(\Omega)$.

In [5] the authors obtained a bound on the intersection number of a geodesic arc on Dome $(\Omega)$ with the bending lamination. (We note that the result is stated in [5] in the setting of domains of discontinuity of Kleinian groups, but the proof goes through readily for general hyperbolic domains, see also [6].) We define $F:\left[0,2 \sinh ^{-1}(1)\right) \rightarrow(0, \infty)$ and $G:(0, \infty) \rightarrow\left[0,2 \sinh ^{-1}(1)\right)$ by setting

$$
F(x)=\frac{x}{2}+\sinh ^{-1}\left(\frac{\sinh (x / 2)}{\sqrt{1-\sinh ^{2}(x / 2)}}\right) \quad \text { and } G(x)=F^{-1}(x) .
$$

Proposition 3.3. ([5, Proposition 5.1]) Let $\Omega \subset \hat{\mathbb{C}}$ be a hyperbolic domain and let $\hat{\nu}: \operatorname{Dome}(\Omega) \rightarrow \mathbb{R}$ be the injectivity radius function on $\operatorname{Dome}(\Omega)$. Let $\mu$ be the bending lamination on $\operatorname{Dome}(\Omega)$. If $\alpha:[0,1) \rightarrow \operatorname{Dome}(\Omega)$ is a geodesic arc of length $l(\alpha) \leq G(\hat{\nu}(\alpha(0))$ which is transverse to $\mu$, then

$$
\mu(\alpha) \leq 2 \pi
$$

Proposition 3.3 may be re-interpreted to give an upper bound on the roundness of the bending lamination of $\operatorname{Dome}(\Omega)$ when $\Omega$ is uniformly perfect.

Proposition 3.4. Let $\Omega \subset \hat{\mathbb{C}}$ be uniformly perfect hyperbolic domain and let $\hat{\nu}>0$ be a lower bound on the injectivity radius of $\operatorname{Dome}(\Omega)$. If $\mu$ is the bending 
lamination of $\operatorname{Dome}(\Omega)$, then

$$
\|\mu\| \leq 2 \pi\left\lceil\frac{1}{G(\hat{\nu})}\right\rceil \leq \frac{4 \pi}{\hat{\nu}}+2 \pi
$$

where $\lceil x\rceil$ is the least integer greater than or equal to $x$.

Proof. Since the function $G$ is increasing, Proposition 3.3, implies that if $\alpha$ is a half open geodesic arc of length at most $G(\hat{\nu})$ which is transverse to $\mu$, then $i(\mu, \alpha) \leq 2 \pi$. Let $\alpha:[0,1) \rightarrow \operatorname{Dome}(\Omega)$ be a unit length geodesic arc which is transverse to $\mu$. We can decompose $\alpha$ into $\lceil 1 / G(\hat{\nu})\rceil$ intervals of length at most $G(\hat{\nu})$. By summing, we see that

$$
i(\alpha, \mu) \leq 2 \pi\left\lceil\frac{1}{G(\hat{\nu})}\right\rceil .
$$

Since $\alpha$ was arbitrary, we conclude that

$$
\|\mu\| \leq 2 \pi\left\lceil\frac{1}{G(\hat{\nu})}\right\rceil \leq \frac{2 \pi}{G(\hat{\nu})}+2 \pi .
$$

If $G(\hat{\nu}) \geq 1$, the final inequality follows immediately. If $G(\hat{\nu})<1$, then $G(\hat{\nu}) \geq \hat{\nu} / 2$, and the final inequality follows from the fact that $\|\mu\| \leq \frac{2 \pi}{G(\hat{\nu})}+2 \pi$.

In [5] we showed that a lower bound on the injectivity radius of $\Omega$ implies an explicit lower bound on the injectivity radius of $\operatorname{Dome}(\Omega)$.

Proposition 3.5. ([5, Lemma 8.1]) If $\Omega \subset \hat{\mathbb{C}}$ is a uniformly perfect domain and $\nu$ is a lower bound for its injectivity radius, then $g(\nu)$ is a lower bound for the injectivity radius of $\operatorname{Dome}(\Omega)$, where

$$
g(\nu)=\frac{e^{-m} e^{-\frac{\pi^{2}}{2 \nu}}}{2} \quad \text { and } \quad m=\cosh ^{-1}\left(e^{2}\right) .
$$

Combining Propositions 3.4 and 3.5 we immediately obtain:

Corollary 3.6. Let $\Omega$ be a uniformly perfect hyperbolic domain and let $\nu>0$ be a lower bound on the injectivity radius of $\Omega$. If $\mu$ is the bending lamination of $\operatorname{Dome}(\Omega)$, then

$$
\|\mu\| \leq 8 \pi e^{m} e^{\frac{\pi^{2}}{2 \nu}}+2 \pi \leq 370 e^{\frac{\pi^{2}}{2 \nu}}+2 \pi
$$


In [6] the authors established that, if $\Omega$ is uniformly perfect, the nearest point retraction lifts to a quasi-isometry between the universal cover $\tilde{\Omega}$ of $\Omega$ and the universal cover $\widetilde{\operatorname{Dome}(\Omega)}$ of $\operatorname{Dome}(\Omega)$.

Theorem 3.7. ([6, Theorem 1.5]) Suppose that $\Omega$ is a uniformly perfect hyperbolic domain and $\nu>0$ is a lower bound for its injectivity radius in the Poincaré metric. Then the nearest point retraction lifts to a quasi-isometry

$$
\tilde{r}: \tilde{\Omega} \rightarrow \widetilde{\operatorname{Dome}(\Omega)}
$$

between the universal cover of $\Omega$ and the universal cover of $\operatorname{Dome}(\Omega)$ where the quasi-isometry constants depend only on $\nu$.

3.3. Complex earthquakes. In this section, we recall the definition of a complex earthquake associated to a lamination (originally introduced by EpsteinMarden [12].) We then describe work of Epstein, Marden and Markovic [14] which uses the theory of holomorphic motions to associate a quasiconformal map to a complex earthquake along a lamination with "small roundness." As a consequence, one obtains a conformally natural quasiconformal map from $\Omega$ to $\operatorname{Dome}(\Omega)$ when the bending lamination of Dome $(\Omega)$ has "small roundness."

If $\mu$ is a measured lamination on $\mathbb{H}^{2}$, one defines the earthquake map

$$
E_{\mu}: \mathbb{H}^{2} \rightarrow \mathbb{H}^{2}
$$

by fixing a component of the complement of $\mu$ and (left-)shearing all other components by an amount given by the measure on $\mu$ (see Thurston [25] or EpsteinMarden [12] for careful discussions). An earthquake map $E_{\mu}$ is continuous except on leaves of $\mu$ with discrete measure (see [14, Section II.3.6]) and extends to a homeomorphism of $S^{1}=\partial \mathbb{H}^{2}$ (see [25, Proposition III.1.2.6]). It follows that a measured lamination $\lambda$ on $\mathbb{H}^{2}$ is mapped to a well-defined measured lamination $E_{\mu}(\lambda)$.

Given a measured lamination $\mu$ and $z=x+i y \in \mathbb{C}$, we define the complex earthquake

$$
\mathbb{C} E_{z}: \mathbb{H}^{2} \rightarrow \mathbb{H}^{3}
$$

to be the result of first earthquaking along $x \mu$ and then bending along $y E_{x \mu}(\mu)$ (see Epstein-Marden [12] for a complete discussion and Epstein-Marden-Markovic $[13$, Section 4] for a discussion in this notation). Formally,

$$
\mathbb{C} E_{z}=P_{y E_{x \mu}(\mu)} \circ E_{x \mu} .
$$


In [13], Epstein, Marden and Markovic find an open neighborhood $\mathcal{T}_{0}$ of the origin such that if $t \in \mathcal{T}_{0}$, the complex earthquake map $\mathbb{C} E_{t}$ extends continuously to $\partial_{\infty} \mathbb{H}^{2}=\mathbb{S}^{1}$ to define a map $\phi_{t}: \mathbb{S}^{1} \rightarrow \hat{\mathbb{C}}$, such that $\phi_{t}\left(\mathbb{S}^{1}\right)$ is a Jordan curve bounding a domain $\Omega_{t}$. They also produce a quasiconformal map $\Phi_{t}: \mathbb{H}^{2} \rightarrow \Omega_{t}$.

Let

$$
f(L, x)=\min \left(\sinh ^{-1}\left(e^{|x|} \sinh L\right), e^{|x| / 2} \sinh L\right)
$$

and define a domain $\mathcal{T}_{0} \subseteq \mathbb{C}$ by

$$
\mathcal{T}_{0}=\left\{x+i y|| y \mid<\frac{c_{2}}{\lceil f(1, x)\rceil}\right\} .
$$

Theorem 3.8. ([13, Theorem 4.14]) Let $\mu$ be a measured lamination on $\mathbb{H}^{2}$ such that $\|\mu\|=1$. Then, for $t \in \mathcal{T}_{0}$, we have

(1) $\mathbb{C} E_{t}$ extends to an embedding $\phi_{t}: \mathbb{S}^{1} \rightarrow \hat{\mathbb{C}}$ which bounds a region $\Omega_{t}$.

(2) If $t=x+i y$ and $y>0$, the bending measure of Dome $\left(\Omega_{t}\right)$ is $y E_{x \mu}(\mu)$.

(3) There is a quasiconformal map $\Phi_{t}: \mathbb{H}^{2} \rightarrow \Omega_{t}$ with dilatation $K_{t}$ given by

$$
K_{t} \leq \frac{1+|h(t)|}{1-|h(t)|}
$$

where $h: \mathcal{T}_{0} \rightarrow \mathbb{H}^{2}$ is a Riemann map taking 0 to 0 .

In particular, $K_{t} \leq 2$ if $|t|<\frac{1}{3}$.

(4) $\Phi_{t} \cup \phi_{t}: \mathbb{H}^{2} \cup \mathbb{S}^{1} \rightarrow \hat{\mathbb{C}}$ is continuous.

(5) If $G$ is a group of Möbius transformations preserving $\mu$, then $\Phi_{t}$ can be chosen so that there is a homomorphism $\rho_{t}: G \rightarrow G_{t}$ where $G_{t}$ is also a group of Möbius transformations and

$$
\Phi_{t} \circ g=\rho_{t}(g) \circ \Phi_{t}
$$

for all $g \in G$.

One obtains the following immediate corollary, whose proof we sketch as a warm-up for the proof of our main result.

Corollary 3.9. (Epstein-Marden-Markovic [14]) If $\Omega \subset \hat{\mathbb{C}}$ is a simply connected, hyperbolic domain, $\mu$ is the bending lamination of $\operatorname{Dome}(\Omega)$ and $\|\mu\|<c_{2}$, then there is a conformally natural $K_{t}$-quasiconformal map $\phi: \Omega \rightarrow \operatorname{Dome}(\Omega)$ which admits a bounded homotopy to the nearest point retraction. 
Proof. Let $y=\|\mu\|$ and $t=i y$. Since $y \in\left(0, c_{2}\right), t \in \mathcal{T}_{0}$. Let

$$
\Phi_{t}: \mathbb{H}^{2} \rightarrow \Omega_{t}
$$

be the $K_{t}$-quasiconformal map provided by Theorem 3.8. Theorem 3.8(2) allows us to conclude that $\Omega_{t}=\Omega$, so

$$
\phi=P_{\mu} \circ \Phi_{t}^{-1}: \Omega \rightarrow \operatorname{Dome}(\Omega)
$$

is a $K_{t}$-quasiconformal map.

Since $\phi$ is quasiconformal, it is a quasi-isometry (see, e.g, [16, Theorem 4.3.2]). Since $\Omega$ is a Jordan domain and the nearest point retraction $r$ and $\phi$ are quasiisometries which agree on $\partial \Omega$, there is a bounded homotopy from $\phi$ to $r$ (see, e.g. [16, Proposition 4.3.1]).

Let $\Gamma$ be a group of Möbius transformations preserving $\Omega$. Then it preserves $\operatorname{Dome}(\Omega)$, and hence its bending lamination. We may pull-back the restriction of the action of $\Gamma$ on $\operatorname{Dome}(\Omega)$, via $P_{\mu}$, to obtain a group $\Gamma^{\prime}$ of conformal automorphisms of $\mathbb{H}^{2}$ which preserve $\mu$. Theorem 3.8(5) implies that we may choose $\Phi_{t}$ so that there exists a homomorphism $\rho_{t}: \Gamma^{\prime} \rightarrow \Gamma_{t}$ so that $\Phi_{t}$ is $\rho_{t}$-equivariant. One may readily check that $\Gamma_{t}$ must agree with $\Gamma$, since they agree on $\partial \Omega$, and hence that $\phi$ is conformally natural.

3.4. Complex angle scaling. Let $\mu$ be a measured lamination on $\mathbb{H}^{2}$ with unit roundness, i.e. $\|\mu\|=1$, and let $t_{0}=i y_{0}$ be a point in $\mathbb{U} \cap \mathcal{T}_{0}$ (where $\mathbb{U}$ is the upper half plane). Let $\Omega_{0}=\Omega_{t_{0}}$ be the domain produced by Theorem 3.8. In [14] Epstein, Marden and Markovic define a map $\Psi: \mathbb{U} \times \Omega_{0} \rightarrow \hat{\mathbb{C}}$ such that each $\Psi_{t}=\Psi(t, \cdot): \Omega_{0} \rightarrow \hat{\mathbb{C}}$ is a locally injective quasiregular map. We recall that a locally injective map $f: \Omega_{0} \rightarrow \hat{\mathbb{C}}$ is $K$-quasiregular if $f=h \circ g$ where $g$ is a $K$-quasiconformal homeomorphism and $h$ is locally injective and holomorphic on the image of $g$. In this section, we describe their construction and its relevant properties.

A key tool in their construction is the family of complex angle scaling maps defined on a crescent. A crescent $C$ is a region bounded by two circles which intersect transversely. Any crescent with interior angle $\theta$ can be mapped by a Möbius transformation to

$$
W_{\theta}=\{z \mid 0 \leq \arg (z) \leq \theta\} .
$$


Given $w \in \mathbb{C}$, we define a complex angle-scaling map on $W_{\theta}$ by setting

$$
S_{w}(z)=z e^{w \arg (z) \theta}
$$

If $(\operatorname{Im}(w)+1) \theta<2 \pi$, then $S_{w}$ is a quasiconformal homeomorphism onto a crescent with interior angle $(\operatorname{Im}(w)+1) \theta$. In general, a complex angle scaling map with domain a crescent $C$ with interior angle $\theta$ has the form $\gamma S_{w} \beta$ for some $w$ where $\gamma$ and $\beta$ are Möbius transformations and $\beta(C)=W_{\theta}$.

Let $\mathcal{N} \subset T_{1}\left(\mathbb{H}^{3}\right)$ denote the set of unit tangent vectors to geodesic rays $\overrightarrow{r(z) z}$ at the point $r(z)$ where $z \in \Omega_{0}$. Informally, $\mathcal{N}$ is the set of normal vectors to $\operatorname{Dome}\left(\Omega_{0}\right)$. Let $\nu: \Omega_{0} \rightarrow \mathcal{N}$ be the obvious homeomorphism. If we let $\exp _{+}: T_{1}\left(\mathbb{H}^{3}\right) \rightarrow \hat{\mathbb{C}}$ be the exponential map which takes a vector to the end point of its corresponding infinite geodesic ray, then $\left.\exp _{+}\right|_{\mathcal{N}}: \mathcal{N} \rightarrow \Omega_{0}$ is the inverse to $\nu$ (see [14, Lemma 4.9]).

For $t \in \mathbb{C}$, they define a map $N_{t}: \mathcal{N} \rightarrow T_{1}\left(\mathbb{H}^{3}\right)$ with the property that if $p$ is the basepoint of a vector $n \in \mathcal{N}$ and $p$ lies in a gap or on a bending line which does not have an atomic weight, then $N_{t}(n)$ is the unique normal vector to the image of $\mathbb{C} E_{t}$ which has the correct orientation. They then define a map $\Psi_{t}: \Omega_{0} \rightarrow \hat{\mathbb{C}}$ by setting $\Psi_{t}(z)=\exp _{+} \circ N_{t} \circ \nu$ if $r_{0}(z)$ lies in a gap or on a bending line which does not have an atomic weight. Otherwise, $r_{0}(z)$ lies in a crescent $r_{0}^{-1}(l)$ in $\Omega_{0}$ which maps to a bending line $l$ with positive measure. On $r_{0}^{-1}(l), \Psi_{t}$ is conjugate to the complex angle scaling map $S_{i\left(t-t_{0}\right) / t_{0}}$. In particular, if $\mu\left(P_{t_{0} \mu}^{-1}(l)\right)=\theta$, $t \in(0, \infty)$ and $t \theta<2 \pi$, then $\Psi_{t}$ is quasiconformal on $r_{0}^{-1}(l)$ and its image is a crescent of total angle $t \theta$ with "endpoints" $\partial \mathbb{C} E_{t}(l)$. The map $\Psi: \mathbb{U} \times \Omega_{t_{0}} \rightarrow \hat{\mathbb{C}}$ is then defined by setting $\Psi(t, z)=\Psi_{t}(z)$.

We summarize their results below:

Theorem 3.10. (Epstein-Marden-Markovic [14, Theorem 4.13]) Let $\mu$ be a measured lamination on $\mathbb{H}^{2}$ such that $\|\mu\|=1$ and let $t_{0}=i y_{0} \in \mathbb{U} \cap \mathcal{T}_{0}$. Then there exists a continuous map $\Psi: \mathbb{U} \times \Omega_{0} \rightarrow \hat{\mathbb{C}}$ such that

(1) The map $\Psi_{t_{0}}=i d$.

(2) For $t \in \mathcal{T}_{0}, \Psi_{t}$ can be extended continuously to a map $\psi_{t}: \partial \Omega_{0} \rightarrow \hat{\mathbb{C}}$ so that $\psi_{t} \circ \phi_{t_{0}}=\phi_{t}$.

(3) For $t \in \mathbb{U} \cap \mathcal{T}_{0}, \Psi_{t}$ is injective and $\Psi_{t}\left(\Omega_{0}\right)=\Phi_{t}\left(\mathbb{H}^{2}\right)=\Omega_{t}$. 
(4) The map $\Psi_{t}$ is a locally injective $L_{t}$-quasiregular mapping, where

$$
L_{t}=\frac{1+|\kappa(t)|}{1-|\kappa(t)|} \quad \text { and } \quad|\kappa(t)|=\left|\frac{t-t_{0}}{t+t_{0}}\right| .
$$

(5) If $G$ is a group of Möbius transformations preserving $\Omega_{0}$, then there is a homomorphism $\rho_{t}: G \rightarrow G_{t}$ where $G_{t}$ is also a group of Möbius transformations, such that

$$
\Psi_{t} \circ g=\rho_{t}(g) \circ \Psi_{t}
$$

for all $g \in G$.

Remark: The equivariance property (5) is not included in the statement of Theorem 4.13 in [14], but is discussed before the proof of Theorem 6.13 in [14]. It follows readily from equivariance properties of complex earthquakes and the explicit description of the map $\Psi$ given above.

\section{Bounds on QUASICONFORMAL DILATATION}

We are now ready to prove our main results. We first provide a sketch of the argument. Given a uniformly perfect domain $\Omega$, there exists a lamination $\mu$ so that $P_{\mu}: \mathbb{H}^{2} \rightarrow \operatorname{Dome}(\Omega)$ is a universal covering map. Let $\mu_{0}=\mu /\|\mu\|$. If we choose $t_{0}=i y_{0} \in \mathcal{T}_{0}$, then Theorem 3.8 provides a $K_{t_{0}}$-quasiconformal map $\Phi_{0}: \mathbb{H}^{2} \rightarrow \Omega_{0}=\Omega_{t_{0}}$. Let $\Psi$ be the map associated to $\mu_{0}$ and $t_{0}$ provided by Theorem 3.10 and let $k=\|\mu\| i$. We use the explicit description of $\Psi$ to check that $\Psi_{k}$ is a $L_{k}$-quasiregular covering map with image $\Omega$. We check that $\Psi_{k} \circ \Phi_{0}$ descends, under the covering map $P_{\mu}$, to a $K_{t_{0}} L_{k}$-quasiconformal map $f: \operatorname{Dome}(\Omega) \rightarrow \Omega$. We then check that $\phi=f^{-1}$ has the desired properties.

Proof of Theorems 1.1 and 1.2: Let $\hat{\mu}$ be the bending lamination of Dome $(\Omega)$. Then $\hat{\mu}$ lifts to give a measured lamination $\mu$ on $\mathbb{H}^{2}$. One may normalize so that $P_{\mu}: \mathbb{H}^{2} \rightarrow \operatorname{Dome}(\Omega)$ is a locally isometric, universal covering map (see Lemma 3.2). Let $G$ be the group of covering transformations of $P_{\mu}$.

Let $k=\|\mu\| i$ and define $\mu_{0}=\frac{1}{|k|} \mu$, so $\mu=|k| \mu_{0}$ and $\left\|\mu_{0}\right\|=1$.

Choose $t_{0}=i y_{0} \in \mathbb{U} \cap \mathcal{T}_{0}$. Theorem 3.8 gives a $K_{t_{0}}$-quasiconformal map

$$
\Phi_{t_{0}}: \mathbb{H}^{2} \rightarrow \Omega_{t_{0}}
$$


whose image is a Jordan domain $\Omega_{t_{0}}$. For simplicity, we denote $\Phi_{0}=\Phi_{t_{0}}$ and $\Omega_{0}=\Omega_{t_{0}}$. Let $\rho_{0}=\rho_{t_{0}}$ be the homomorphism provided by Theorem 3.8 and let $G_{0}=\rho_{0}(G)$. Let $r_{0}: \Omega_{0} \rightarrow \operatorname{Dome}\left(\Omega_{0}\right)$ be the nearest point retraction. Notice that

$$
P_{0}=P_{t_{0} \mu_{0}}: \mathbb{H}^{2} \rightarrow \operatorname{Dome}\left(\Omega_{0}\right)
$$

is an isometry which extends continuously to a map $\phi_{0}: \mathbb{S}^{1} \rightarrow \hat{\mathbb{C}}$ and that

$$
\Phi_{0} \cup \phi_{0}: \mathbb{H}^{2} \cup \mathbb{S}^{1} \rightarrow \hat{\mathbb{C}}
$$

is continuous (see Lemma 3.2 and Theorem 3.8). Then

$$
F_{0}=P_{0} \circ \Phi_{0}^{-1}: \Omega_{0} \rightarrow \operatorname{Dome}\left(\Omega_{0}\right)
$$

is a conformally natural $K_{t_{0}}$-quasiconformal homeomorphism which extends to the identity on $\partial \Omega_{0}$ (see Corollary 3.9).

Let $\Psi: \mathbb{U} \times \Omega_{0} \rightarrow \hat{\mathbb{C}}$ be the map associated to $\mu_{0}$ and $t_{0}$ provided by Theorem 3.10. Consider the map $\Psi_{k}: \Omega_{0} \rightarrow \hat{\mathbb{C}}$. Notice that $\mathbb{C} E_{k}=P_{\mu}$. So, by the definition of $\Psi$, if $p$ lies in a component of $\mathbb{H}^{2}-\mu$ or on a bending line which does not have atomic weight, then $\Psi_{k}\left(r_{0}^{-1}\left(P_{0}(p)\right)=r^{-1}\left(P_{\mu}(p)\right)\right.$. Moreover, if $l$ is a leaf with atomic weight, then $\Psi_{k}$ restricts to a quasiconformal homeomorphism from $r_{0}^{-1}\left(P_{0}(l)\right)$ to $r^{-1}\left(P_{\mu}(l)\right)$ given by a complex angle scaling map between the two crescents.

Therefore, $\Psi_{k}$ is a conformally natural, locally injective $L_{k}$-quasiregular map with image $\Omega$. Moreover, $\Psi_{k}$ is a topological universal covering map with covering transformations $G_{0}$.

The composition

$$
F=\Psi_{k} \circ \Phi_{0}: \mathbb{H}^{2} \rightarrow \Omega
$$

is a $K_{t_{0}} L_{k}$-quasiregular universal covering map with covering transformations $G$. Therefore, it descends, under the covering map $P_{\mu}: \mathbb{H}^{2} \rightarrow \operatorname{Dome}(\Omega)$, to a $K_{t_{0}} L_{k}$-quasiconformal homeomorphism

$$
f: \operatorname{Dome}(\Omega) \rightarrow \Omega
$$




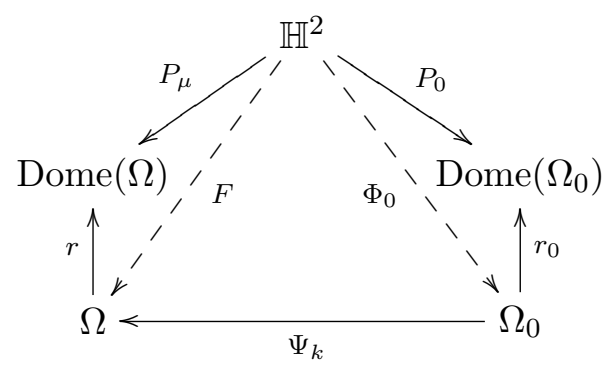

We claim that $f$ is a homotopy inverse for $r$ and that there is a bounded homotopy between $\phi=f^{-1}$ and $r$. Consider the conformal universal covering map $p: \tilde{\Omega} \rightarrow \Omega$ of $\Omega$. Let $\tilde{G}$ denote the group of covering transformations of the universal covering map $p$. The quasiregular map $F$ lifts to a quasiconformal map $\tilde{F}: \mathbb{H}^{2} \rightarrow \tilde{\Omega}$ which conjugates the action of $G$ to the action of $\tilde{G}$. The maps $\tilde{F}$ and $\Phi_{0}$ are quasi-isometries, since they are quasiconformal (see, e.g. [16, Theorem $4.3 .2])$.

Let $l$ be a geodesic in $\mathbb{H}^{2}$ which is either disjoint from $\mu$ or is a leaf of $\mu$. Let $a$ and $b$ be the endpoints of $l$. Then $\Phi_{0}(l)$ is a quasigeodesic with endpoints $\phi_{0}(a)$ and $\phi_{0}(b)$. Let $A_{l}=r_{0}^{-1}\left(P_{0}(l)\right)$. Since there is a bounded homotopy between $F_{0}$ and $r_{0}$, there is a bounded Hausdorff distance between $\Phi_{0}(l)$ and $A_{l}$. In particular, $\Phi_{0}(l)$ and $A_{l}$ accumulate at the same endpoints at infinity. By construction

$$
\Psi_{k}\left(A_{l}\right)=r^{-1}\left(P_{k}(l)\right)=B_{l}
$$

and $\Psi_{k}$ is a complex angle scaling map on $A_{l}$. Therefore, $F(l)$ is a quasigeodesic which lies a bounded Hausdorff distance from $B_{l}$.

Recall that $P_{\mu}: \mathbb{H}^{2} \rightarrow \operatorname{Dome}(\Omega)$ is a locally isometric covering map, so this gives an explicit identification of the universal cover $\widetilde{\operatorname{Dome}(\Omega)}$ of $\operatorname{Dome}(\Omega)$ with $\mathbb{H}^{2}$. With this identification, $\tilde{F}: \mathbb{H}^{2} \rightarrow \tilde{\Omega}$ is a lift of $f: \operatorname{Dome}(\Omega) \rightarrow \Omega$. Fix a leaf $l_{0}$ of $\mu$. We may then choose a lift $\tilde{r}: \tilde{\Omega} \rightarrow \widetilde{\operatorname{Dome}(\Omega)}=\mathbb{H}^{2}$ so that $\tilde{r}\left(\tilde{F}\left(l_{0}\right)\right)$ is a quasigeodesic which is a bounded Hausdorff distance from $l_{0}$. With this normalization, if $l$ is any geodesic which either lies in $\mu$ or is disjoint from $\mu$, then $\tilde{r}(\tilde{F}(l))$ is a quasigeodesic which is a bounded Hausdorff distance from $l$. Since every point in $\mathbb{S}^{1}$ is an endpoint of such a geodesic and $\tilde{r} \circ \tilde{F}$ is a quasi-isometry (being a composition of quasi-isometries), we see that $\tilde{r} \circ \tilde{F}$ extends to the identity on $\mathbb{S}^{1}$ and the straight-line homotopy between $\tilde{r} \circ \tilde{F}$ and the identity map on $\mathbb{H}^{2}$ is a bounded homotopy (see, e.g. [16, Proposition 4.3.1]). It follows, since $\tilde{F}$ is a quasi-isometry and a homeomorphism, that the straight-line homotopy between $\tilde{r}$ 
and $\tilde{F}^{-1}$ is also a bounded homotopy. This homotopy descends to give a bounded homotopy between $r$ and $\phi=f^{-1}$.

Let $\Gamma$ be the group of conformal automorphisms of $\Omega$. $\Gamma$ is also a group of conformal automorphisms of $\operatorname{Dome}(\Omega)$ which preserves the bending lamination. Let $\hat{\Gamma}$ be the group of all conformal automorphisms of $\mathbb{H}^{2}$ which arise as lifts, under the covering map $P_{\mu}$, of restrictions of elements of $\Gamma$ to $\operatorname{Dome}(\Omega)$. Notice that $G \subset \hat{\Gamma}$ and that $\hat{\Gamma}$ preserves $\mu$. Theorems 3.8 and 3.10 insure that we may assume that there exist homomorphisms $\rho_{0}: \hat{\Gamma} \rightarrow \hat{\Gamma}_{0}$ and $\rho_{k}: \hat{\Gamma}_{0} \rightarrow \hat{\Gamma}_{k}$ so that $\Phi_{0}$ is $\rho_{0}$-equivariant and $\Psi_{k}$ is $\rho_{k}$-equivariant. Notice that $\rho_{0}(G)=G_{0}, \rho_{k}\left(G_{0}\right)$ is trivial, $\rho_{k}\left(\hat{\Gamma}_{0}\right)=\Gamma$, and $F: \mathbb{H}^{2} \rightarrow \Omega$ is $\left(\rho_{k} \circ \rho_{0}\right)$-equivariant. Since the action of $\hat{\Gamma}$ on $\mathbb{H}^{2}$ descends, via $P_{\mu}$, to the action of $\Gamma$ on $\operatorname{Dome}(\Omega)$, it follows that $f$, and hence $\phi$, is conformally natural.

Therefore, $\phi$ is a conformally natural $K_{t_{0}} L_{k}$-quasiconformal map from $\Omega$ to $\operatorname{Dome}(\Omega)$ which admits a bounded homotopy to the nearest point retraction. It only remains to check the claimed bounds on the quasiconformal dilatation.

Theorem 3.10 implies that

$$
L_{k}=\frac{\|\mu\|}{y_{0}}
$$

SO

$$
K_{t_{0}} L_{k}=\frac{K_{t_{0}}}{y_{0}}\|\mu\|
$$

We can choose $y_{0} \in(0,1 / 3)$ arbitrarily close to $1 / 3$ in which case we can choose $K_{t_{0}}=2$, so we may assume that

$$
K_{t_{0}} L_{k} \leq 6\|\mu\| \text {. }
$$

Applying Proposition 3.4 and Corollary 3.6, which give bounds on $\|\mu\|$ in terms of the injectivity radii of $\Omega$ and $\operatorname{Dome}(\Omega)$, we obtain

$$
K_{t_{0}} L_{k} \leq 6\left(\frac{4 \pi}{\hat{\nu}}+2 \pi\right)=N(\hat{\nu})
$$

and

$$
K_{t_{0}} L_{k} \leq 6\left(8 \pi e^{m} e^{\frac{\pi^{2}}{2 \nu}}+2 \pi\right)=M(\nu) .
$$

It follows that $\phi$ is $M(\nu)$-quasiconformal and $N(\hat{\nu})$-quasiconformal as claimed, which completes the proof. 


\section{Round ANnUli}

Let $\Omega(s)$ denote the round annulus lying between concentric circles of radius 1 and $e^{s}>1$ about the origin. Then $\Omega(s)$ has conformal modulus $s / 2 \pi$. (Recall that if $A \subset \hat{\mathbb{C}}$ is a hyperbolic domain which is homeomorphic to an annulus, then there exists a unique $s_{A}>1$ such that $A$ is conformal to $\Omega\left(s_{A}\right)$ and one defines its conformal modulus to be $\bmod (A)=s_{A} / 2 \pi$.) In the Poincaré metric $\Omega(s)$ is a complete hyperbolic annulus with core curve of length

$$
\frac{2 \pi^{2}}{s}=\frac{\pi}{\bmod (\Omega(s))} \text {. }
$$

In its intrinsic metric, Dome $(\Omega(s))$ is a complete hyperbolic annulus with core curve of length $\frac{2 \pi}{\sinh \left(\frac{s}{2}\right)}$. In particular, Dome $(\Omega(s))$ has modulus

$$
\bmod (\operatorname{Dome}(\Omega(s)))=\sinh (s / 2) / 2 .
$$

Therefore, the minimal quasiconformal dilatation $K(s)$ of a quasiconformal map between $\Omega(s)$ and $\operatorname{Dome}(\Omega(s))$ is given by

$$
K(s)=\frac{\pi \sinh (s / 2)}{s} .
$$

The injectivity radius bounds are given by

$$
\nu(s)=\nu(\Omega(s))=\frac{\pi^{2}}{s} \quad \text { and } \quad \hat{\nu}(s)=\hat{\nu}(\Omega(s))=\frac{\pi}{\sinh (s / 2)} .
$$

Therefore, as $s \rightarrow \infty$ and $\nu \rightarrow 0$,

$$
K(s)=\frac{\nu(s) \sinh \left(\frac{\pi^{2}}{2 \nu(s)}\right)}{\pi} \approx \frac{\nu(s) e^{\frac{\pi^{2}}{2 \nu(s)}}}{2 \pi}=O\left(\frac{M(\nu(s))}{\log (M(\nu(s)))}\right)
$$

and

$$
K(s)=\frac{\pi^{2}}{2 \hat{\nu}(s) \sinh ^{-1}(\pi / \hat{\nu}(s))} \approx \frac{\pi^{2}}{2 \hat{\nu}(s) \log (1 / \hat{\nu}(s))}=O\left(\frac{N(\hat{\nu}(s))}{\log (N(\hat{\nu}(s)))}\right) .
$$

\section{General LOWer Bounds on the Quasiconformal COnStant}

In this section, we give an explicit lower bound on the dilatation of a quasiconformal map from $\Omega$ to $\operatorname{Dome}(\Omega)$ which admits a bounded homotopy to the nearest point retraction. As in the last section, our lower bound has "almost" the same asymptotics as the upper bound in our main theorem. 
Proposition 6.1. Let $\Omega$ be a uniformly perfect domain in $\hat{\mathbb{C}}$. Suppose that $\phi: \Omega \rightarrow \operatorname{Dome}(\Omega)$ is a $K$-quasiconformal map which is homotopic to the nearest point retraction. If $\Omega$ contains a point of injectivity radius $\nu \in(0, .5)$, in the Poincaré metric, then

$$
K \geq \frac{\nu e^{\frac{\pi^{2}}{2 \sqrt{e} \nu}}}{\pi^{2} e^{\frac{\pi}{2}}}=O\left(\nu e^{\frac{\pi^{2}}{2 \sqrt{e} \nu}}\right)
$$

Proof. Let $\gamma$ be a simple closed geodesic in $\Omega$ with length $L \leq 2 \nu$. Theorem 5.1 in [10] implies that $r(\gamma)$ is homotopic to a closed geodesic $r(\gamma)^{*}$ in $\operatorname{Dome}(\Omega)$ with length

$$
L^{\prime}<\frac{4 \pi e^{.502 \pi}}{e^{\frac{\pi^{2}}{\sqrt{e} L}}}<.153 .
$$

Maskit [19, Proposition 1] and Sugawa [22, Theorem 5.2] showed that if $\alpha$ is a simple closed geodesic of length $l$ in a hyperbolic surface $X$ and $M(\alpha)$ is the maximal modulus of an annulus in $X$ whose core curve is homotopic to $\alpha$, then

$$
\frac{\pi}{l} \geq M(\alpha) \geq \frac{\pi}{l e^{l / 2}} .
$$

Therefore, $r(\gamma)^{*}$ is homotopic to the core curve of an annulus $A^{\prime}$ in $\operatorname{Dome}(\Omega)$ with modulus

$$
\bmod \left(A^{\prime}\right) \geq \frac{\pi}{L^{\prime} e^{\frac{L^{\prime}}{2}}}>\frac{2}{L^{\prime}}
$$

Since $\phi$ is $K$-quasiconformal, $A=\phi^{-1}\left(A^{\prime}\right)$ has modulus

$$
\bmod (A) \geq \frac{\bmod \left(A^{\prime}\right)}{K} \geq \frac{2}{K L^{\prime}}
$$

On the other hand, since the core curve of $A$ is homotopic to $\gamma$,

$$
\bmod (A) \leq \frac{\pi}{L}
$$

so

$$
K \geq \frac{2 L}{\pi L^{\prime}} \geq \frac{L e^{\frac{\pi^{2}}{\sqrt{e} L}}}{2 \pi^{2} e^{.502 \pi}} \geq \frac{\nu e^{\frac{\pi^{2}}{2 \sqrt{e} \nu}}}{\pi^{2} e^{\frac{\pi}{2}}}
$$

as claimed. (In the final inequality we use the fact that $h(\nu)=\nu e^{\frac{\pi^{2}}{2 \sqrt{e} \nu}}$ is decreasing on the interval $(0, .5)$.) 


\section{REFERENCES}

[1] L. Bers, "On boundaries of Teichmüller spaces and Kleinian groups I," Annals of Math. 91(1970), 570-600.

[2] C.J. Bishop, "Divergence groups have the Bowen property," Annals of Math. 154(2001), 205-217.

[3] C.J. Bishop, "An explicit constant for Sullivan's convex hull theorem," in In the Tradition of Ahlfors and Bers III, Contemp. Math. 355(2007), Amer. Math. Soc., 41-69.

[4] M. Bridgeman, "Average bending of convex pleated planes in hyperbolic three-space," Invent. Math. 132(1998), 381-391.

[5] M. Bridgeman and R.D. Canary, "From the boundary of the convex core to the conformal boundary," Geom. Ded. 96(2003), 211-240.

[6] M. Bridgeman and R.D. Canary, "The Thurston metric on hyperbolic domains and boundaries of convex hulls," G.A.F.A. 20(2010), 1317-1353.

[7] M. Bridgeman, R.D. Canary and A. Yarmola, in preparation.

[8] J. Brock, R.D. Canary and Y. Minsky, "The classification of Kleinian surface groups II: the ending lamination conjecture," Annals of Math., 176(2012), 1-149.

[9] R.D. Canary, "The Poincaré metric and a conformal version of a theorem of Thurston," Duke Math. J. 64(1991), 349-359.

[10] R.D. Canary, "The conformal boundary and the boundary of the convex core," Duke Math. J. 106(2001), 193-207.

[11] A. Douady and C. Earle, "Conformally natural extensions of homeomorphisms of the circle," Acta. Math. 157(1986), 23-48.

[12] D.B.A. Epstein and A. Marden, "Convex hulls in hyperbolic space, a theorem of Sullivan, and measured pleated surfaces," in Analytical and Geometrical Aspects of Hyperbolic Space, Cambridge University Press, 1987, 113-253.

[13] D.B.A. Epstein, A. Marden and V. Markovic, "Quasiconformal homeomorphisms and the convex hull boundary," Annals of Math. 159(2004), 305-336.

[14] D.B.A. Epstein, A. Marden and V. Markovic, "Complex earthquakes and deformations of the unit disk," J. Diff. Geom 73(2006), 119-166.

[15] D.B.A. Epstein and V. Markovic, "The logarithmic spiral: a counterexample to the $K=2$ conjecture," Annals of Math. 161(2005), 925-957.

[16] A. Fletcher and V. Markovic, Quasiconformal Maps and Teichmüller Theory, Oxford Graduate Texts in Mathematics, 2007.

[17] Y. Komori and C. Matthews, "An explicit counterexample to the equivariant $K=2$ conjecture." Conform. Geom. Dyn. 10(2006), 184-196.

[18] A. Marden and V. Markovic, "Characterizations of plane regions that support quasiconformal mappings to their domes," Bull. L.M.S. 39(2007), 962-972.

[19] B. Maskit, "Comparison of hyperbolic and extremal lengths," Ann. Acad. Sci. Fenn. 10(1985), 381-386.

[20] Y. Minsky, "The classification of Kleinian surface groups I: models and bounds," Annals of Math., 171(2010), 1-107. 
[21] C. Pommerenke, "On uniformly perfect sets and Fuchsian groups," Analysis 4(1984) 299321.

[22] T.Sugawa, "Various domain constants related to uniform perfectness," Comp. Var. Th. and Appl. 36(1998), 311-345.

[23] D.P. Sullivan, "Travaux de Thurston sur les groupes quasi-fuchsiens et les variétés hyperboliques de dimension 3 fibrées sur $S^{1}$," in Bourbaki Seminar, Vol. 1979/1980, Lecture Notes in Math. 842(1981), 196-214.

[24] W.P. Thurston, The Geometry and Topology of 3-Manifolds, Lecture Notes, Princeton University, 1979, available at: http://www.msri.org/publications/books/gt3m/

[25] W. P. Thurston, "Earthquakes in two-dimensional hyperbolic geometry," in Lowdimensional Topology and Klienian, Cambridge University Press, 1986, 91-112.

[26] W. P. Thurston, "Hyperbolic structures on 3-manifolds, II: surface groups and 3-manifolds which fiber over the circle," preprint, available at: http://front.math.ucdavis.edu/math.GT/9801045

\section{Martin Bridgeman}

Department of Mathematics

Boston College

E-mail: bridgem@bc.edu

Richard D. Canary

Department of Mathematics

University of Michigan

E-mail: canary@umich.edu 